\title{
Chronic Evaluation of a Clinical System for Deep Brain Stimulation and Recording of Neural Network Activity
}

\author{
Paul H. Stypulkowski Scott R. Stanslaski Timothy J. Denison \\ Jonathon E. Giftakis \\ Medtronic Neuromodulation, Minneapolis, Minn., USA
}

\section{Key Words}

Hippocampal deep brain stimulation • Epilepsy · Chronic recording $\cdot$ Neural activity

\begin{abstract}
Background/Aims: In conjunction with therapeutic stimulation, next-generation deep brain stimulation (DBS) devices may offer the ability to record and analyze neural signals, providing for unprecedented insight into DBS effects on neural networks. This work was conducted to evaluate an implantable, clinical-grade system that permits concurrent stimulation and recording using a large animal (ovine) model recently developed to study DBS for epilepsy. Methods: Following anesthesia and 1.5-tesla MRI acquisition, unilateral anterior thalamic and hippocampal DBS leads were implanted $(n=3)$ using a frameless stereotactic system. Chronic, awake recordings of evoked potentials (EPs) and local field potentials were collected with the implanted device and analyzed off-line. Results: Hippocampal EPs were stable over long-term ( $>1$ year) recording and consistent in morphology and latency with prior acute results. Thalamic and hippocampal DBS produced both excitatory and inhibitory network effects that were stimulation site and parameter dependent. Free roaming recordings illustrated periods of
\end{abstract}

\begin{tabular}{ll}
\hline KARGER & $\begin{array}{l}\text { ( } 2013 \text { S. Karger AG, Basel } \\
1011-6125 / 13 / 0914-0220 \$ 38.00 / 0\end{array}$ \\
E-Mail karger@karger.com & $\begin{array}{l}\text { This is an Open Access article licensed under the terms } \\
\text { of the Creative Commons Attribution-NonCommercial- } \\
\text { www.karger.com/sfn }\end{array}$ \\
$\begin{array}{l}\text { NoDerivs 3.0 License (www.karger.com/OA-license), appli- } \\
\text { cable to the online version of the article only. Distribution } \\
\text { for non-commercial purposes only. }\end{array}$
\end{tabular}

highly correlated activity between these two structures within the circuit of Papez. Conclusions: These results provide further insight into mechanisms of DBS therapy for epilepsy and an encouraging demonstration of the capabilities of this new technology, which in the future, may afford unique opportunities to study human brain function and neuromodulation mechanism of action.

Copyright $\odot 2013$ S. Karger AG, Basel

\section{Introduction}

Deep brain stimulation (DBS) has emerged as an effective therapy for treatment-resistant movement disorders including Parkinson's disease, essential tremor, and dystonia [1]. The clinical success in these indications has encouraged investigations of this therapy modality to treat multiple other disorders, including epilepsy, obsessive compulsive disorder, depression, and Alzheimer's disease [2-7]. Many of the brain targets in use today are based upon historical neurosurgical lesion sites, while others are hypothesis driven and grounded in current un-

All authors are employees of Medtronic Inc.
Paul H. Stypulkowski, PhD

Medtronic Neuromodulation

7000 Central Avenue NE

Minneapolis, MN 55432 (USA)

E-Mail paul.stypulkowski@medtronic.com 
derstanding of neural networks. Stimulation parameters and patterns employed across indications have generally been empirically derived, and based upon clinical observations, when possible.

The mechanism of action of DBS remains unclear, and may potentially involve a number of related cellular and network processes given the different time courses of effect observed between disorders. Studies have suggested that different oscillatory patterns within brain networks may reflect states of normal function [8-10], or conversely reflect pathological states associated with symptoms of specific neurological disease [11-13]. A leading hypothesis regarding DBS mechanism of action involves the disruption of such pathological oscillations, releasing the network to resume more normal function. Supporting evidence for this theory comes from intraoperative [14, 15] or temporary postoperative [16] recordings of local field potentials (LFPs) from implanted DBS leads.

Currently approved DBS devices can deliver electrical stimulation to multiple electrodes over a wide range of stimulus parameters. However, unlike implantable devices used for cardiac therapies (e.g. pacemakers and defibrillators), which can directly measure and report the effects of stimulation on the target organ, the present generation of neurostimulators does not have the capability to record physiologic signals from implanted leads. We report here the first chronic evaluation, in a large animal model, of a new DBS hardware platform designed to permit long-term recording of electrical brain activity [17], and to thereby directly examine the effects of DBS on specific neural networks.

This work was conducted in an ovine model recently developed in our laboratory to study DBS for epilepsy [18]. The prior study investigated stimulation and recording from nodes within the circuit of Papez, specifically the anterior nuclear (AN) region of the thalamus, and the hippocampus (HC). A series of preclinical studies have suggested that this limbic circuit is involved in the generation and propagation of seizure activity [19-23]. In addition, a series of pilot studies [24-27] and a recently completed randomized controlled trial [28], have demonstrated that anterior thalamic DBS can be effective in reducing seizures in patients.

Based upon our earlier findings from acute recordings within this neural circuit in anesthetized animals, the work described here was undertaken to assess the ability to chronically stimulate and record, using a fully implanted clinical-grade system, from DBS electrodes in this brain network. Specifically, the goals of the current study were to: (a) determine whether it was possible to record evoked potentials (EPs) and LFPs from these neural structures over extended periods of time, (b) assess the stability of these recordings, and (c) compare the results of DBS in this network in awake animals with those reported in the prior study. In addition, this new system afforded the opportunity to chronically record LFPs simultaneously from the thalamus and the $\mathrm{HC}$ in awake, free-roaming animals, and to evaluate the interactions between these structures during various brain states.

\section{Methods}

This study was conducted at the Physiological Research Laboratory (Medtronic Inc., Minneapolis, Minn., USA) under a protocol approved by the institutional Animal Care and Use Committee.

\section{Surgery}

Adult Polypay mixed-breed sheep (acute $\mathrm{n}=1$; chronic $\mathrm{n}=3$, average weight $53 \mathrm{~kg}$ ) were anesthetized with a standard regimen: morphine as premedication, followed by induction with propofol, intubated and maintained on $>2 \%$ isoflurane, with a fentanyl/ ketamine drip. Following anesthesia, 1.5-tesla MRIs were collected and transferred to a surgical planning station (Stealth Station Treon; Medtronic Inc.) where trajectories for unilateral thalamic and hippocampal DBS leads were calculated based upon gross anatomic descriptions of the ovine brain [29] and the prior study experience. Leads were implanted using a frameless stereotactic system (NexFrame) following similar procedures used for human DBS surgery [30].

Following implantation of the HC lead (model 3387; $1.5-\mathrm{mm}$ contacts, $1.5-\mathrm{mm}$ spacing), intraoperative stimulation to confirm thalamic lead (model 3389; $1.5-\mathrm{mm}$ contacts, $0.5-\mathrm{mm}$ spacing) placement was delivered to the most distal contact pair as the lead was advanced in 1-mm steps along the planned trajectory. Hippocampal EPs and LFPs were recorded using standard Grass EEG amplifiers and digitized with the BioPac MP150 system (BioPacSystems Inc., Goleta, Ga., USA). Based upon these electrophysiologic measures, final lead position was determined, the thalamic lead locked into the burr hole cap, and extensions connected to both leads and tunneled to a postscapular pocket. The extensions were connected to the modified Activa PC neurostimulator in the thoracic pocket. In an acute pilot animal (A1), a series of EP and LFP recordings were collected using the standard laboratory equipment and then repeated using the implantable device to assess the in vivo stimulation and recording capabilities. In the chronic subjects (C1-3), following closure of all incisions, anesthesia was discontinued, and the animals transferred to surgical recovery. Postoperative $\mathrm{CT}$ images were collected 13 weeks after implant $(\mathrm{C} 1)$ or immediately after surgery $(\mathrm{C} 2, \mathrm{C} 3)$. These scans were later merged with preoperative $\mathrm{MR}$ images to assess the approximate stereotactic location of the DBS leads.

\section{Stimulation and Recording}

Following a 2-week recovery period, recording sessions were conducted weekly for the first 3 months, and monthly thereafter, for approximate 2-hour periods with the animals awake and rest- 
ing in a sling. The implanted system allowed for stimulation and recording from both leads, with specific configurations selected via programmable telemetry interfaces. Recording parameters (sampling rate, filter cutoffs, center frequency, bandwidth, etc.) for time domain signals and power channels were set via a custom-designed programming interface tablet. The power channel functionality provided the ability to tune a recording channel to a specific frequency band and to record energy fluctuations occurring within that band. Stimulation parameters for the device were controlled using the standard DBS Physician Programmer (model 8840).

Electrode impedances and hippocampal EPs in response to thalamic stimulation were collected at each session. For EP measurements, trains of stimuli at $5 \mathrm{~Hz}$ were presented for $30 \mathrm{~s}$ at levels of 2-7 V (120- $\mu$ s pulse width). Initially, each adjacent pair of contacts on the thalamic lead was tested to determine the cathode/anode combination that produced the largest hippocampal EPs. This bipolar pair was then used for subsequent long-term EP testing, and to evaluate stimulation effects on HC LFPs with highfrequency stimulus bursts. Similarly, for the EP recordings, the contact pair on the HC lead that yielded the maximal evoked response amplitude was determined, and then used for all chronic recordings (specific recording configuration for each animal is detailed in figure legends). Stimulation of the HC lead at various parameters was also performed to assess both local and network effects. All EPs and LFPs were recorded by the implanted device (422-Hz sampling rate; $0.5-\mathrm{Hz} \mathrm{HP}, 100-\mathrm{Hz}$ LP filters), downloaded, and analyzed off-line.

After completing each stimulation/recording session, data from the implanted devices were downloaded, the memory cleared, and the devices programmed to operate in the loop recording mode. Loop recordings were triggered in two ways, either on a timed schedule, or based on an algorithm programmed to recognize specific patterns of neural activity. This device capability provided the opportunity to record LFPs from both leads simultaneously throughout the day, under different behavioral and environmental conditions. Using a $200-\mathrm{Hz}$ sampling rate, approximately $55 \mathrm{~min}$ of time domain LFP activity from two channels could be recorded before the memory was full. Data from these free roaming loop recordings were then downloaded at the next scheduled session and analyzed off-line.

All recordings involving stimulation were conducted with the subjects resting in a sling, which provided easy access to the device for stimulation parameter changes and observation of any potential stimulation-induced adverse effects. The timed/triggered loop recordings (no stimulation) were collected with the animals free roaming in their home environment.

\section{Data Analyses}

Postoperative CT scans were merged with preoperative MRI images on the Stealth Station, and the stereotactic location of the DBS lead contacts calculated relative to the mid-commissural point. Electrophysiologic data were analyzed using Acqknowledge 4.1 software (BioPac Systems). EPs were averaged off-line using the stimulus artifact as the trigger. Spectral analysis of the LFPs was conducted with Sigview32 software (v2.3.0). Spectrograms (intensity of instantaneous frequency vs. time; Hann window) are displayed using a logarithmic Z-axis with color representing relative intensity (heat palette; black = low, white/ yellow $=$ high).

\section{Results}

\section{Surgical Targeting}

Consistent with the earlier study [18], it was possible to reliably target and implant clinical-grade DBS leads into the anterior thalamic and hippocampal regions in this species using MRI-based, frameless stereotactic techniques. For each animal, the specific thalamic cathodal contact that generated the largest HC EPs was determined, and its stereotactic location identified on preoperative MR images. Figure 1 shows a representative set of MRIs from one subject (C1), illustrating this point in multiple stereotactic planes. Despite known inaccuracies associated with lead artifact estimation and image fusion, this location was consistent in all subjects with the anterior thalamic region, and corresponded well with results of the same analysis performed in the prior study. Placement of DBS leads into the $\mathrm{HC}$, a large, easily visualized cortical structure, was highly repeatable with the methods employed.

\section{Thalamic Stimulation - EPs}

Figure 2a shows a series of EPs recorded intraoperatively from the $\mathrm{HC}$ in response to stimulation of the thalamic lead as it was introduced along the planned trajectory and advanced in 1-mm steps. In this animal, the evoked response amplitude reached a maximum at $2 \mathrm{~mm}$ past the nominal target, and then declined with further lead advancement. This response pattern was used to determine the final lead position for all subjects.

Figure $2 \mathrm{~b}$ shows a series of awake EPs recorded from this same subject, approximately 4 weeks after surgery, using the fully implanted device for both stimulation and recording. As observed in the prior study, the HC EPs show a clearly graded, amplitude-dependent input-output response with a latency in the range of approximately $40 \mathrm{~ms}$. These chronic recordings were also consistent in both morphology and latency with the intraoperative responses. Figure 3 illustrates the chronic HC EPs recorded with the implanted devices over various periods of time from each of the three subjects (C1 - 64 weeks, C2 - 52 weeks, C3 - 40 weeks; follow-up times vary due to staggered implant dates). For all of these recordings, the stimulus parameters were fixed $(5 \mathrm{~V}, 120 \mu \mathrm{s}, 5 \mathrm{~Hz})$ to assess the stability of the responses over time. In each case, the within-subject EPs appeared quite stable, with consistent amplitude, morphology, and latency over long periods of time. As expected, the response morphology and amplitude between subjects did vary somewhat, based upon small differences in $\mathrm{HC}$ lead position and re- 
Fig. 1. DBS lead location. Stereotactic position of the cathode on the thalamic lead that generated the largest hippocampal EPs (subject C1) shown in coronal (a), sagittal (b), and axial (c) planes. d This location transposed to a mid-sagittal image illustrating position relative to the midline thalamus. For reference, AC-PC length is $13.7 \mathrm{~mm}$.
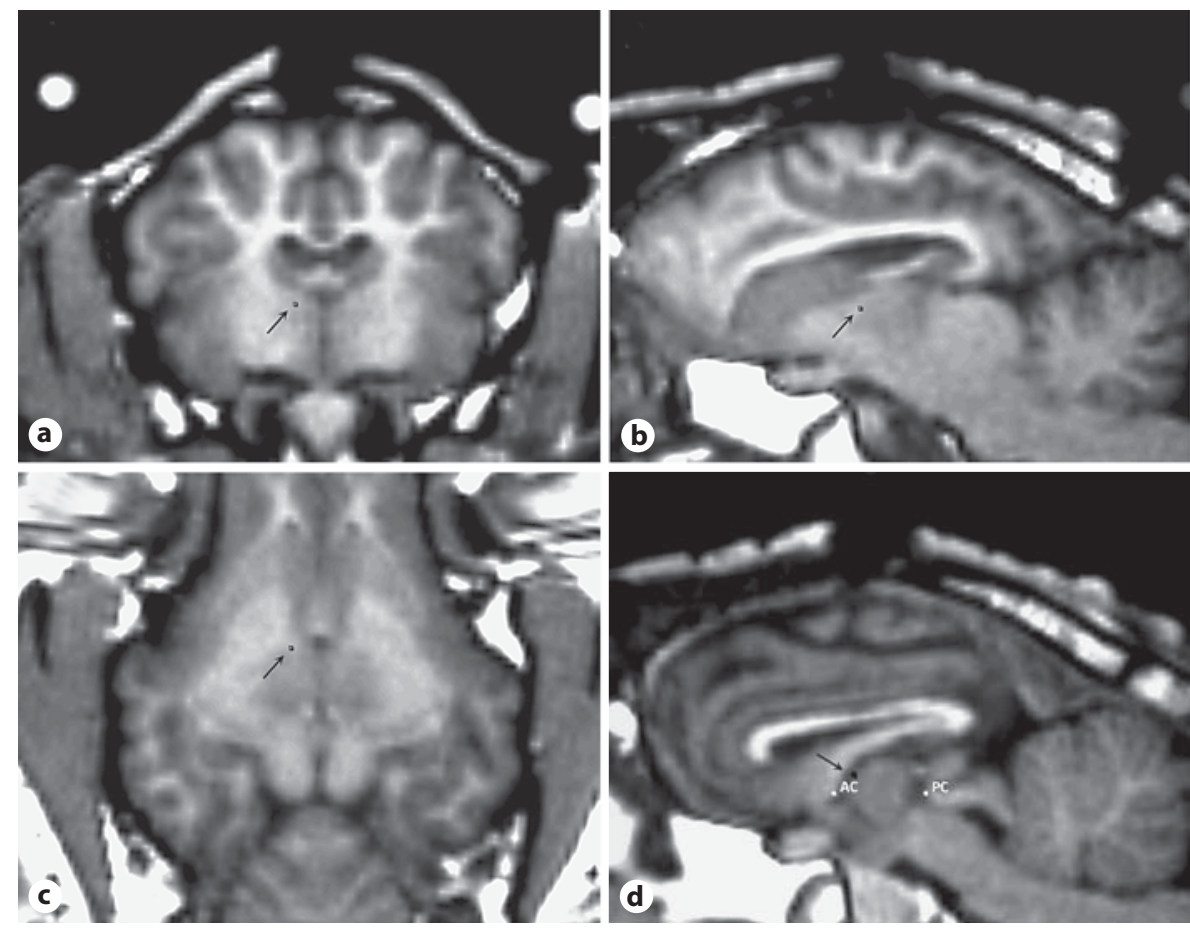

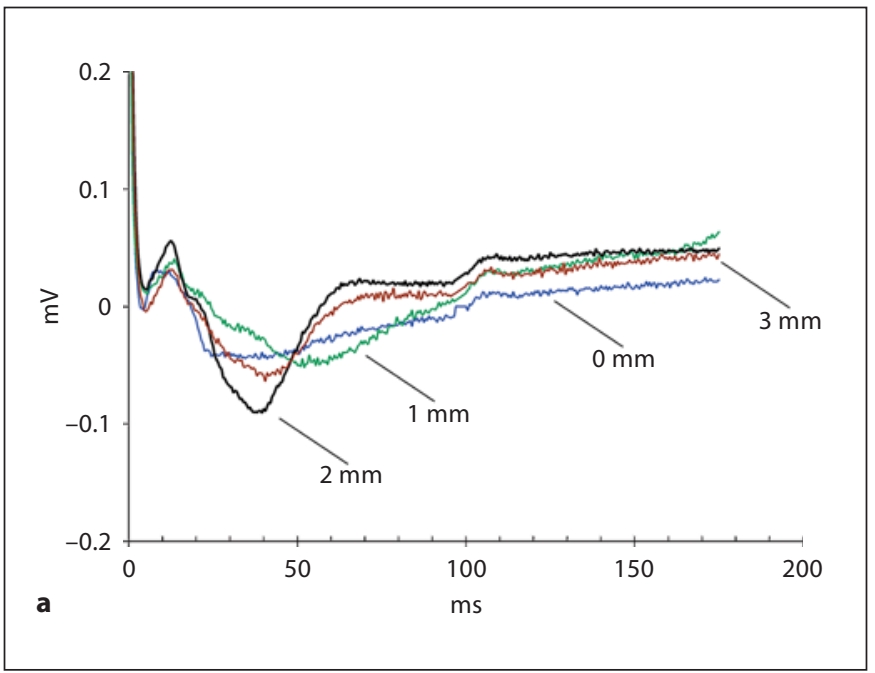

Fig. 2. Hippocampal EPs. a Intraoperative EPs recorded in response to bipolar stimulation $(0-1+, 7 \mathrm{~V}, 120 \mu \mathrm{s}, 5 \mathrm{~Hz})$ at different thalamic lead positions along the planned trajectory (subject $\mathrm{C} 1$ ); maximal EP response was generated at a position of $2 \mathrm{~mm}$ past nominal target; stimulation and recording with laboratory hard-

cording contact configuration relative to the actual source dipole of the EP. Also, due to use of the stimulus artifact as the averaging trigger, and sampling rate limitations of the implanted device, the absolute EP latencies could vary over several milliseconds between recordings.

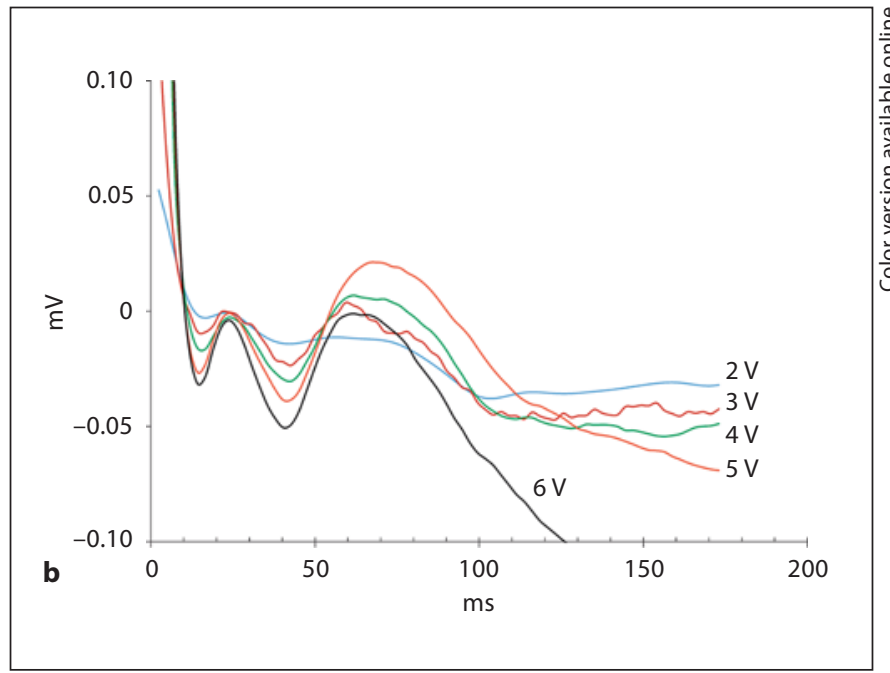

ware. b Chronic EPs from the same animal 4 weeks after surgery illustrating graded input-output response to different stimulus amplitudes; stimulation and recording with the implanted device $(2-1+, 120 \mu \mathrm{s}, 5 \mathrm{~Hz})$.

\section{Thalamic Stimulation - LFPs}

An interesting observation from the previous study in anesthetized animals was the pronounced suppression of hippocampal LFPs induced by high-frequency anterior thalamic stimulation. We examined that effect in this 


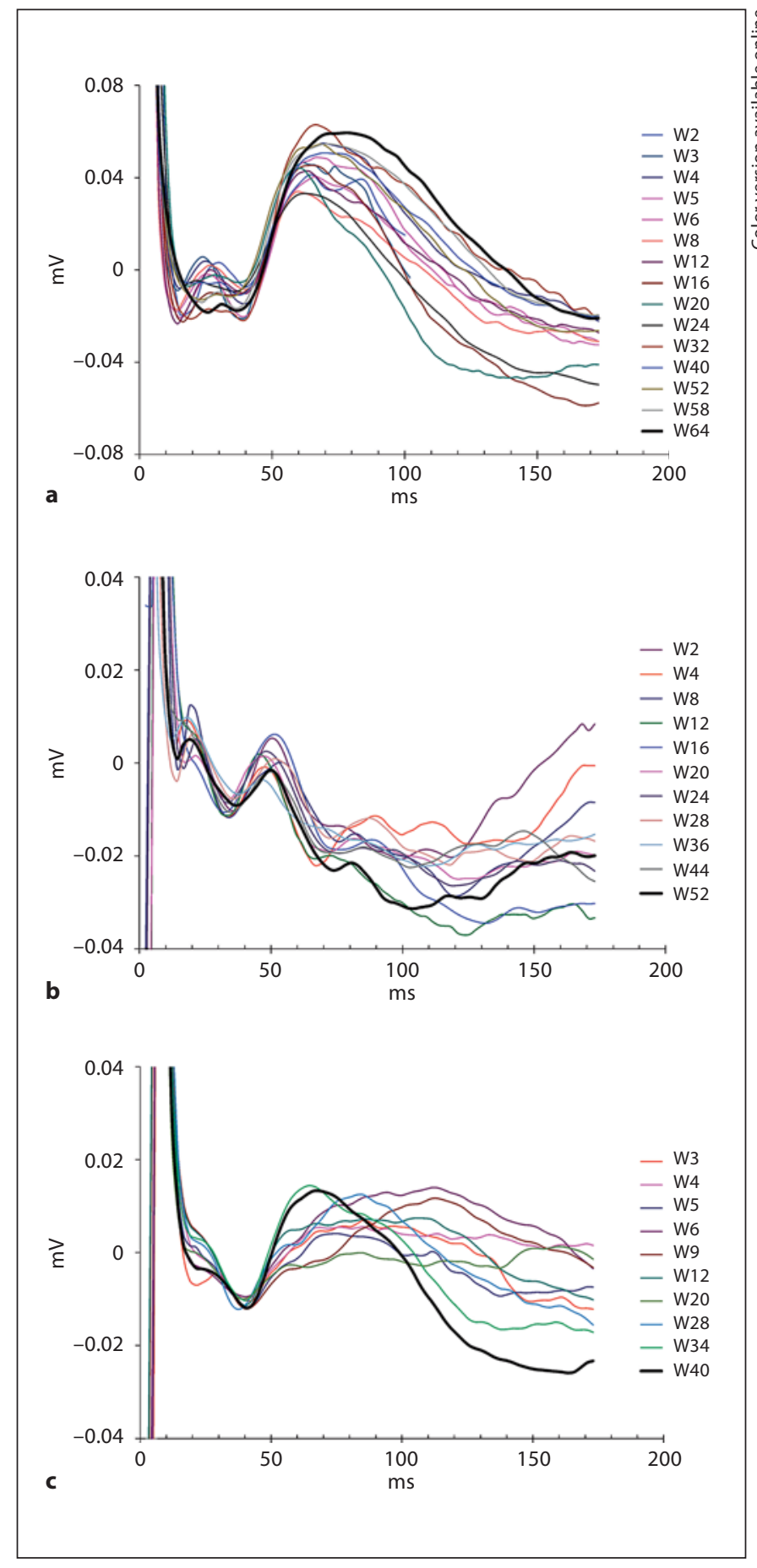

Fig. 3. Chronic hippocampal EPs illustrating long-term stability. Evoked responses recorded at multiple time points (weeks after implant as listed) from the three subjects. Subject C1 (a): recording electrodes - HC03, stimulation contacts - AN2-1+; subject C2 (b): recording electrodes - $\mathrm{HC13}$, stimulation contacts - AN1-2+; subject C3 (c): recording electrodes - HC01, stimulation contacts - AN2-1+. Stimulation parameters (5 V, $120 \mu \mathrm{s}, 5 \mathrm{~Hz})$ were held constant for all recordings. The bold trace in each panel represents the response measured at the longest follow-up time. study, both intraoperatively and chronically in awake animals. Figure 4 shows two series of spectrograms from intraoperative recordings which demonstrate the targeting specificity associated with this effect. Figure 4 a shows thalamic (top channel) and HC LFPs (bottom three channels) recorded while a series of stimulus bursts, at different frequencies, was applied to a pair of contacts on the thalamic lead. The stimulus artifact can be seen in the thalamic channel; however, there is little effect of the stimulation observed in the hippocampal spectrograms. In contrast, the recordings in figure $4 \mathrm{~b}$ show the effects of the same set of stimuli applied to the adjacent pair of thalamic contacts, which shifted the center of the active cathode by $2 \mathrm{~mm}$. In this case, there was a pronounced suppression of neural activity across all frequency bands induced by high-, but not low-frequency stimulus bursts, observed in two of the three hippocampal channels. Interestingly, the third $\mathrm{HC}$ channel exhibited a very 'high Q' local 2-Hz oscillation evoked by the stimulation, which lasted for several minutes, and subsided in parallel with the return of the spontaneous activity on the other channels. Of note, the thalamic LFP activity mirrored that of the HC in both cases, with little change in the first example, and in the second, showing a reduction in activity coincident with the time course of changes observed in the HC. A corresponding low-frequency oscillation was also observed in the thalamic recording (and was most pronounced on a different channel, which is not shown).

The spectrograms in figure 5 represent HC LFPs collected from each of the three subjects during awake recording sessions and illustrate the effects of high-frequency thalamic stimulation under these conditions. These LFPs show a dominant band of theta activity in the $\mathrm{HC}$ that is absent in the anesthetized recordings. Each panel shows a recording of several minutes that contains a series of repeated high-frequency stimulus bursts applied to the thalamic lead. During the on-period of the stimulation, the HC LFP activity is suppressed, but in contrast to the anesthetized condition, returns to baseline levels very rapidly after the end of the stimulus burst, with little carryover effect. The overall level of suppression across the frequency spectrum also appears reduced, compared to the intraoperative example. It should be noted, however, that the intraoperative stimulation amplitudes were greater than those used during the awake recordings, which were limited by behavioral side effects.

\section{Hippocampal Stimulation - LFPs}

Direct stimulation of the hippocampal lead was also evaluated in these animals. Depending upon the level and 


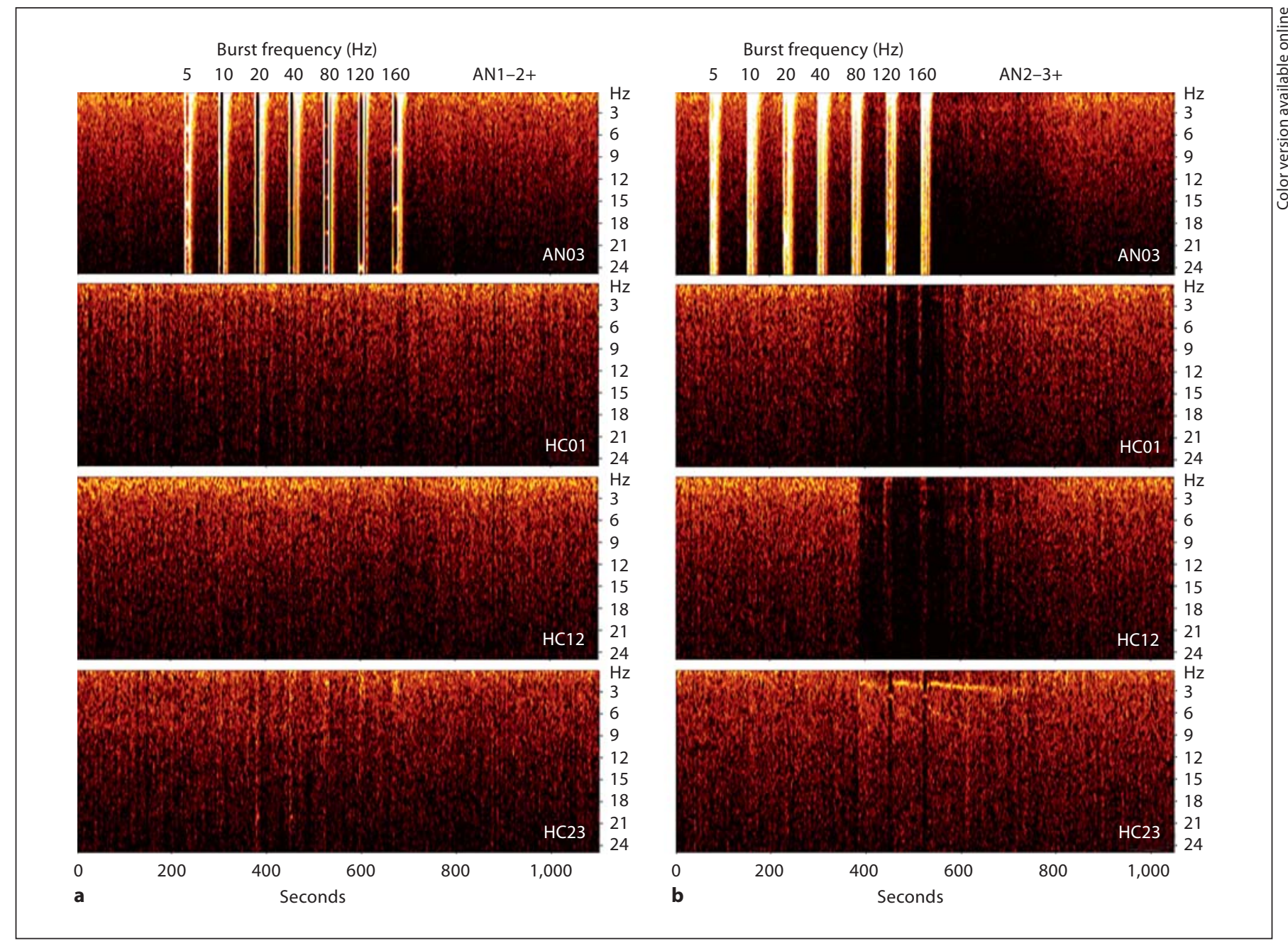

Fig. 4. Spectrograms of intraoperative LFPs recorded from the thalamus (top channel) and HC (bottom three channels; subject A1) in response to thalamic stimulation. Recording electrode pairs are shown for each channel. a Stimulation was applied to thalamic contacts $1-2+$ at different frequencies shown $(7 \mathrm{~V}, 120$ $\mu \mathrm{s}, 10$-second bursts, 1 burst per min). Stimulus artifact in the top panel denotes periods of stimulation, which had no apparent effects on LFP activity. $\mathbf{b}$ The same set of stimuli applied to thalamic contacts $2-3+$, which resulted in suppression of LFP activity with high-frequency stimulation. frequency of stimulation applied, a range of effects on HC LFPs was observed. In contrast to thalamic stimulation, direct stimulation of the HC could result in the generation of local epileptiform discharges, whose characteristics were dependent upon the stimulation parameters. In some cases, these appeared as classically described afterdischarges which began following a short burst of stimulation and then self-terminated. In other cases, typically with high-frequency stimulation (e.g. $>100 \mathrm{~Hz}$ ), the discharge began during the stimulus burst and then terminated as the stimulation ended, or shortly thereafter. Notably, at levels that were below threshold to elicit these discharges, stimulation often produced a clear suppression of local activity, which lasted for some period of time after the stimulus burst.

Figure 6 provides an illustration of these different effects and the dependence upon stimulus parameters. Panel a shows the time domain signal and corresponding spectrogram of a HC LFP recorded over a period of approximately $15 \mathrm{~min}$. Three short bursts of $50 \mathrm{~Hz}$ stimulation were applied to the hippocampal lead at increasing amplitude levels. The first, lowest amplitude stimulus $(0.5 \mathrm{~V})$ was sub-threshold for any effect, which is most easily seen in the spectrogram (again note the 


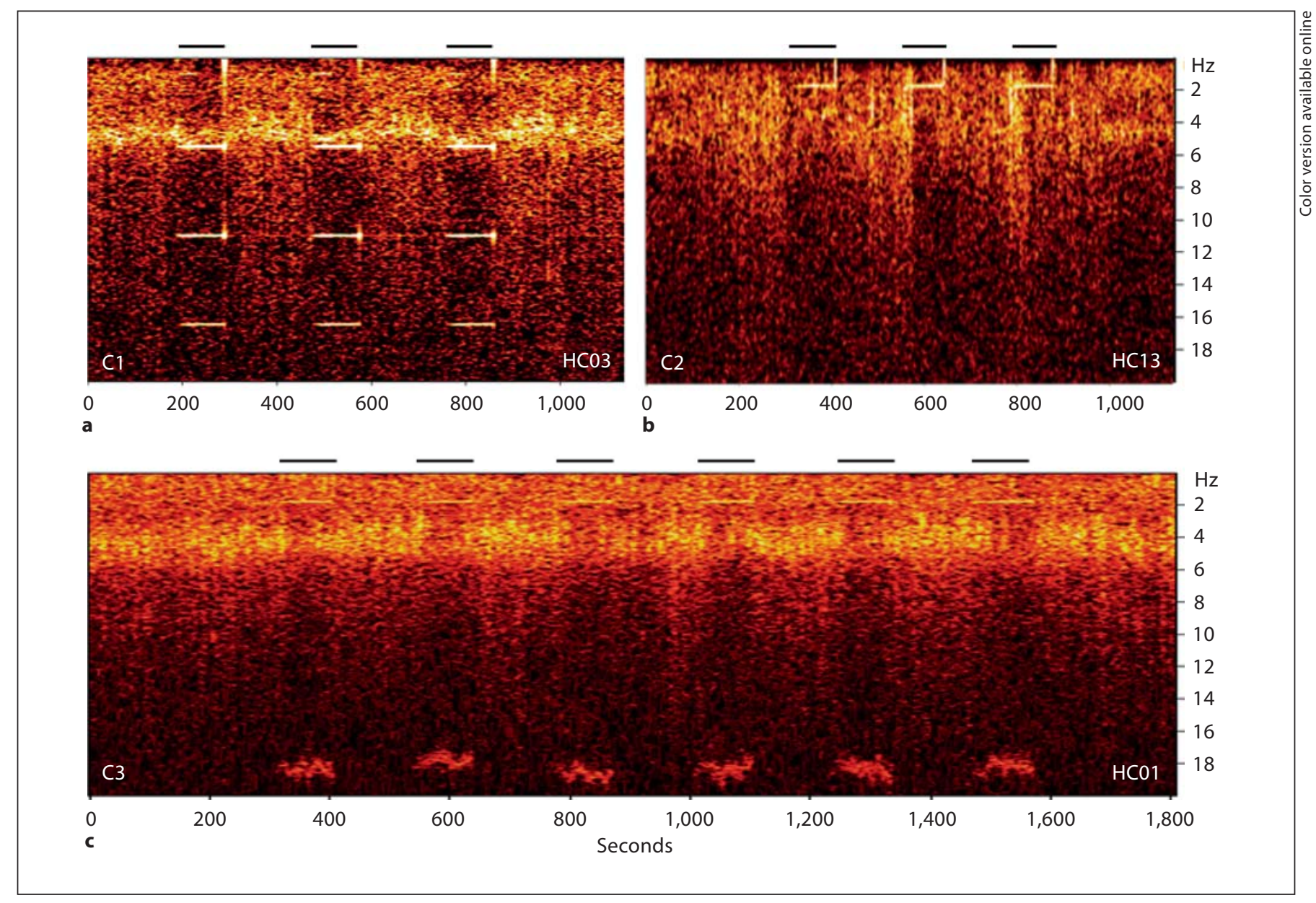

Fig. 5. Spectrograms of hippocampal LFPs recorded from 3 awake subjects. Thalamic stimulation was cycled on and off for repeated 60 -second periods (indicated by the bars above each panel), which resulted in reduced $\mathrm{HC}$ activity during stimulation. a Subject $\mathrm{C1}$ : 4.0 V, 3 cycles. b Subject C2: 4.5 V, 3 cycles. c Subject C3: 5.0 V, 6 cycles. Stimulation contacts $2-1+, 120 \mu \mathrm{s}, 105 \mathrm{~Hz}$ for all examples. Recording electrode pairs are shown for each subject (records from subjects $\mathrm{C} 1$ and $\mathrm{C} 3$ show stimulus frequency harmonics associated with an early firmware version). dominant $\mathrm{HC}$ theta band activity in the awake recording). The second stimulus $(0.9 \mathrm{~V})$ resulted in an immediate reduction of LFP activity, apparent in both the time domain signal as well as the spectrogram, which persisted for several minutes. Finally, the highest amplitude stimulus $(1.3 \mathrm{~V})$ resulted in the generation of an afterdischarge, which lasted for approximately $30 \mathrm{~s}$, and was followed by a prolonged period of suppressed local activity. During the afterdischarge, the animal exhibited a stereotypical orienting behavioral response. A second recording in this same subject (panel b) illustrates the influence of stimulus frequency on the local inhibitory effect. In this case, the stimulation amplitude was fixed at $0.9 \mathrm{~V}$, and stimulus bursts of different frequencies were presented. As the burst frequency was increased, the reduction in LFP activity became apparent at frequencies of $20 \mathrm{~Hz}$ and greater. Unlike the example shown in figure 4 with thalamic stimulation, this reduction in $\mathrm{HC}$ activity was not reflected in the simultaneous AN recording (not shown).

\section{Free Roaming Recordings}

Figure 7 illustrates examples of some of the neural activity patterns that were observed during the free roaming, timed loop recordings. Panels $a_{1}$ and $a_{2}$ show thalamic and HC LFPs from the same animal that were captured $4 \mathrm{~h}$ apart during the early morning (lights out) hours. The top traces show the anterior thalamic record- 


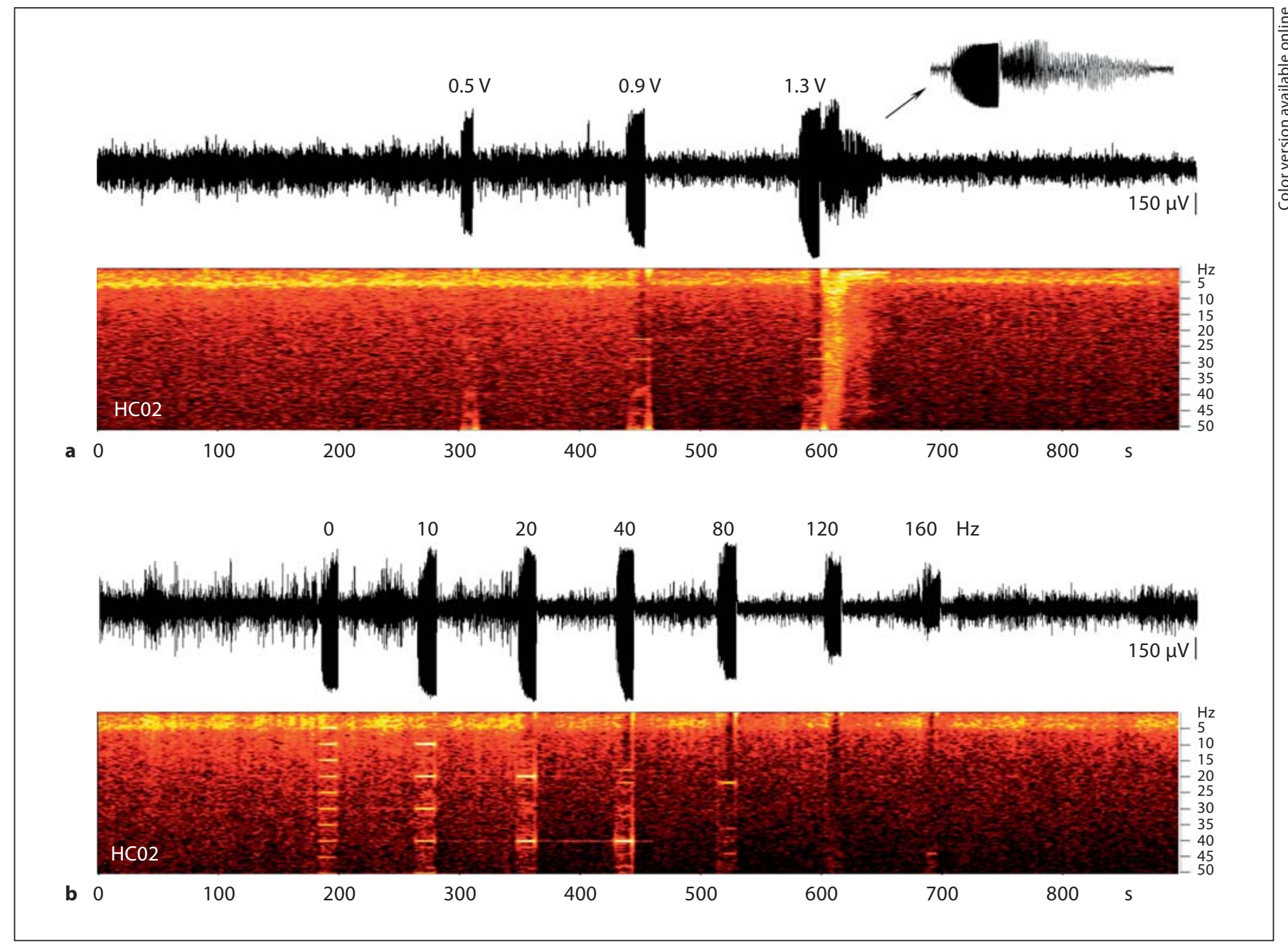

Fig. 6. Effects of direct hippocampal stimulation on LFPs (subject C1). a Amplitude effects. Time domain signal (top) and spectrogram (bottom) showing the effects of short bursts of stimulation (1-C+, $300 \mu \mathrm{s}, 50 \mathrm{~Hz}, 5$-second burst with ramped onset) at increasing amplitudes $(0.5 \mathrm{~V}$ - no effect; $0.9 \mathrm{~V}$-suppression; $1.3 \mathrm{~V}$ afterdischarge). Inset shows the final stimulus and the generated afterdischarge on an expanded time scale. b Frequency effects. Time domain signal (top) and spectrogram (bottom) illustrating the effects of short bursts of stimulation at different frequencies (1-C+, $0.9 \mathrm{~V}, 300 \mu \mathrm{s}, 5$-second burst with ramped onset). Suppression of $\mathrm{HC}$ activity was observed with stimulus frequencies of 20 $\mathrm{Hz}$ and above. ings which contain large amplitude spikes, not seen in the $\mathrm{HC}$ channel, that are consistent with the appearance of $\mathrm{K}$ complexes [31], typically observed during sleep. Each K complex is preceded by a lower amplitude spike approximately 6-7 s earlier. The left and right panels suggest two clearly different brain states, with both structures relatively inactive in panel $\mathrm{a}_{1}$, and with notably increased network activity in panel $\mathrm{a}_{2}$. Panel $\mathrm{b}$ shows a recording from a different subject which contains a series of characteristic hippocampal theta bursts, but no apparent correlated activity in the thalamus.
Figure 8 provides examples of both the timed and triggered recording capability of the implanted device. Each panel shows time domain signals and corresponding power channel energy estimates of thalamic and HC LFP recordings. Panel a (top) was captured as a timed recording, and included a period of high amplitude, low-frequency hippocampal sharp wave (SPW) activity with a coincident increase in thalamic firing. The power channels clearly show the correlation in activity between these two nodes within this neural circuit. Using this SPW pattern as a template, the device's on-board algorithm was 


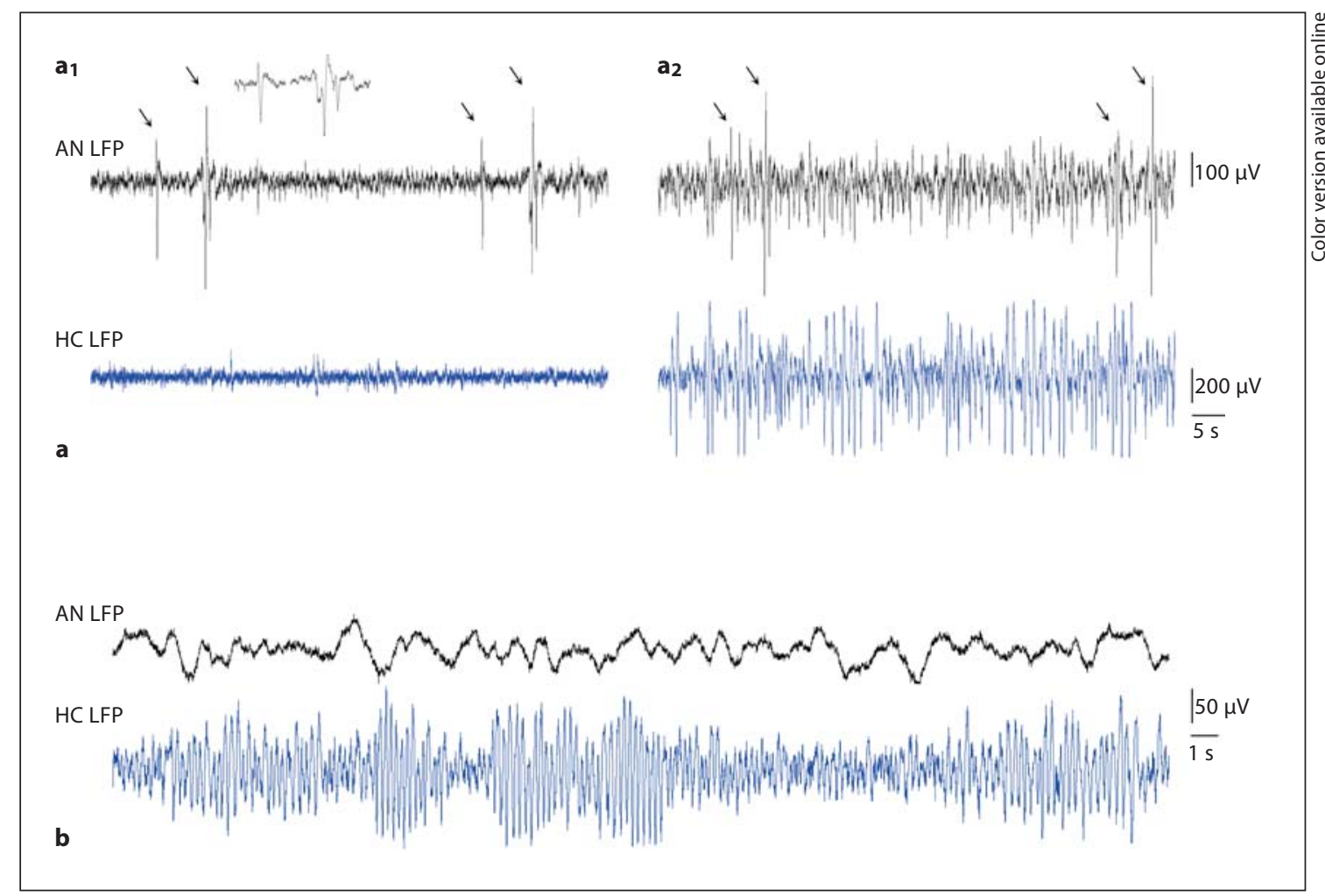

Fig. 7. Free roaming recordings illustrating different network states. a Sequential timed recordings $\left(\mathbf{a}_{\mathbf{1}}-1\right.$ am; $\mathbf{a}_{\mathbf{2}}-5 \mathrm{am}$ ) from subject C2 showing thalamic (top) and hippocampal (bottom) LFPs. Arrows indicate K complexes and associated spikes in thalamic recordings. $\mathbf{a}_{\mathbf{1}}$ Inset shows these discharges in greater detail. $\mathbf{b}$ Thalamic (top) and hippocampal (bottom) LFP recordings from subject C3 illustrating multiple theta bursts in the HC.

programmed to detect similar patterns of activity, and to trigger a loop recording, which included a 30-second preevent buffer. Panel b (bottom) shows one of the events captured during this type of triggered, free roaming recording, illustrating a similar burst of HC SPWs and a corresponding increase in thalamic network activity.

\section{Discussion}

The primary objective of this study was to evaluate the chronic in vivo stimulation and recording capabilities of a new DBS hardware platform. In that regard, the results of this work were highly encouraging. With the exception of a single electrode contact that became intermittent shortly after implant (subject C2), the recording system generally performed as expected over three cumulative 'subject' years of experience, and comparable to traditional laboratory hardware, given anticipated limitations.
Several points of comparison are worthy of comment. First, it should be noted that the implanted device recordings are true differential measures rather than traditional referential, grounded recordings. As such, some of the long-standing electrophysiologic assumptions regarding these signals needed to be reconsidered. For example, in a standard referential EP recording, reversing the electrical stimulus polarity causes the stimulus artifact to invert. In these floating differential measurements, however, this was not always observed. These recordings are highly sensitive to the spatial orientation of the closely spaced recording bipole and source dipole. In addition, the common mode coupling of stimulation circuit to the sensing channel, within the implant, often dominates over external tissue effects during the actual stimulation pulse.

Although the recovery time of the implanted device amplifiers was expectedly slower than rack-mounted systems, it was more than adequate for recording the EPs of 
Fig. 8. Timed and triggered free roaming recordings. a Timed recording from subject C2 showing LFPs and power channel energy estimates from the thalamus (top pair of traces) and HC (bottom pair of traces) illustrating sharp wave activity. b Triggered recording from the same subject using event detection algorithm programmed to detect similar patterns of hippocampal activity as recorded in $\mathbf{a}$.

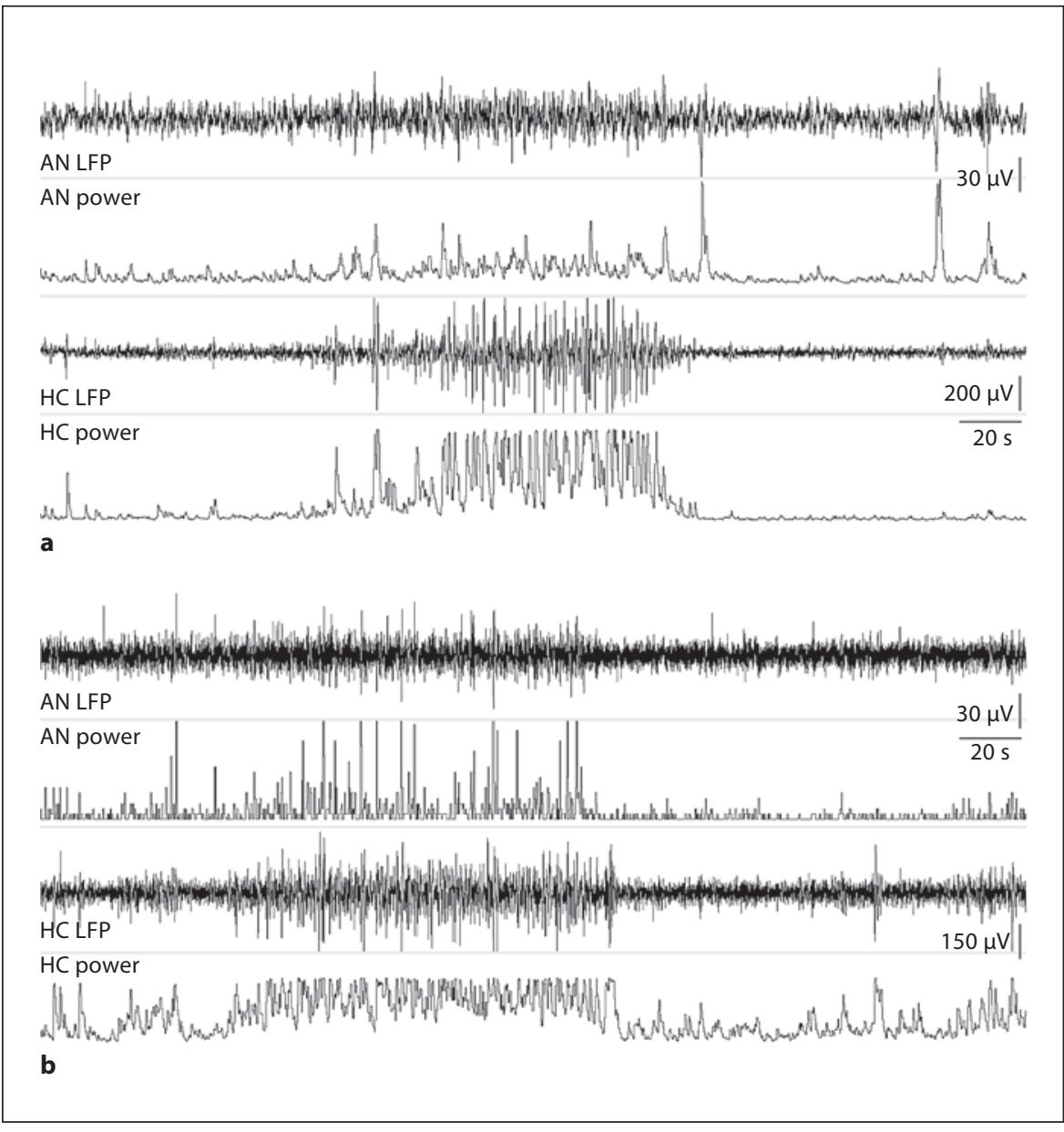

interest in this study. As seen in figure 2, the recovery time with the laboratory amplifiers was on the order of 2-3 ms, and was approximately $10 \mathrm{~ms}$ for the implanted device. Another important performance characteristic of the implanted system was the common mode rejection, which allowed recording of LFPs during stimulation, even on the same lead, without the use of amplifier blanking circuits. This is illustrated in figures 5 and 6 which show examples of HC spectrograms during both thalamic and HC stimulation. In both cases, spectrally relevant information was preserved in the frequency domain, as the stimulation frequencies were much higher than the LFP bands of interest. The ability to record LFP activity on the same lead during the delivery of stimulation required a specific configuration: monopolar stimulation (case anodal), and symmetrical recording electrodes around the stimulation contact (e.g. stimulation 1-, C+; recording contacts 0 and 2). This configuration resulted in maximal common mode rejection of the stimulus ar- tifact and produced the least interference in the background LFP signal. In all of these recordings, however, it was required that the gain was at a level that avoided artifact-induced amplifier saturation. If any amplifier saturation occurred, the information in both the time and frequency domain signals was corrupted, leading to potentially erroneous conclusions about physiological effects of stimulation.

From the physiologic perspective, this study provided a number of interesting insights, some of which warrant further study. The characteristics of the EPs (latency, morphology, input-output response) recorded in the awake, chronically implanted animals were entirely consistent with the description reported in our prior acute study, and demonstrated long-term stability in all 3 subjects. Electrode impedances did show some variability from session to session, which was not surprising, given that the leads were not chronically stimulated. However, the long-term within-subject reproducibility of the EPs 
suggests that either small differences in impedance were negligible, even with a constant voltage system, or that stimulation of the contacts resulted in a rapid stabilization of impedance to a similar level at each session [32]. The consistency of the EPs is also indicative of a stable physiologic system, with no apparent ongoing changes associated with implantation of the DBS leads.

One key observation in this study was the difference between the effects of thalamic stimulation on the $\mathrm{HC}$ LFPs in the awake versus anesthetized animals. In the prior acute study, and in the intraoperative recordings during this study (fig. 4), marked suppression of hippocampal activity, which far outlasted the stimulus, was observed in response to high-frequency thalamic stimulation. These results have been consistently seen in anesthetized animals under two different anesthetic regimens. In contrast, the suppression observed in the awake animals was not nearly as pronounced, nor as long lasting. One factor that may have contributed to this observation was the level of stimulation. The stimulus amplitudes delivered during the awake recordings were considerably lower than those tested during the anesthetized recordings. Stimulation at high frequencies was limited by behavioral side effects in the awake animals, which generally consisted of motor responses or apparent disorientation. These maximum tolerated levels were always lower than those that produced the maximal awake EP (at $5 \mathrm{~Hz}$ ), but within the voltage range where HC EPs were elicited. Even with the higher levels used in the anesthetized animals, however, there was a clear specificity to the HC suppression, as illustrated in figure 4, suggesting that stimulation was activating a relatively restricted neural population, and was not attributable to a generalized, non-specific effect.

The data of figure 4 also indicate that this suppression was due to the influence of thalamic stimulation on the $\mathrm{HC}$ and associated network, and not a local inhibitory effect in the thalamus. In the first example, stimulation produced no change in HC or thalamic LFP activity. However, simply moving the active cathode to an adjacent contact resulted in marked HC suppression, which was then presumably reflected back into the thalamus via the Papez circuit. The reduction in thalamic activity occurred only in the presence of HC suppression, suggesting the thalamic change was due to network, and not local, effects. The appearance on one HC channel of a stimulation-induced, highly-tuned, local oscillation that persisted for several minutes, and its reflection in the thalamic recording (at a slightly lower frequency) was an interesting phenomenon and was also observed in one other animal.
In the awake animals, the HC LFPs exhibited a dominant band of theta activity, which was not present in the anesthetized animals. The balance between excitatory and inhibitory drive within this network is likely very different under these two behavioral conditions. It is possible that in the presence of reduced excitatory activity and upregulated inhibitory drive, characteristic of the anesthetized state, a strong, prolonged inhibitory effect from high-frequency thalamic stimulation was revealed. In contrast, similar stimulation in the awake animal yielded a reduced level of hippocampal suppression, perhaps due to a more excitatory bias in the network. With respect to thalamic DBS for epilepsy, this raises interesting clinical questions, including how stimulation may interact with various classes of antiepileptic drugs.

Direct stimulation of the $\mathrm{HC}$ in the awake subjects revealed an amplitude- and frequency-dependent pattern of results. The occurrence of afterdischarges in response to $\mathrm{HC}$ stimulation was expected based upon the extensive description of this phenomenon in the literature $[33,34]$. Some of these discharges had the characteristics of classically described ADs as shown in figure 6, while others appeared to be driven only during the stimulation burst. A more unexpected result was the appearance of HC suppression at stimulus levels just below the threshold for $\mathrm{AD}$ generation. This LFP inhibition was highly reproducible and could be obtained within a narrow window of stimulation levels that were supra-threshold for this effect but sub-threshold for $\mathrm{AD}$ generation. The reduction in spontaneous activity persisted for some time after the end of stimulation, and exhibited a clear frequency dependence. Interestingly, the frequency threshold $(20 \mathrm{~Hz})$ for this locally generated inhibitory effect was much lower than the frequency required to produce hippocampal suppression via thalamic stimulation $(80 \mathrm{~Hz})$. However, the observation that the suppression resulting from direct $\mathrm{HC}$ stimulation was not reflected in the network activity recorded at the thalamus suggests that it may be a relatively localized effect, consistent with the low stimulation amplitude used.

This set of results in particular highlights the capability provided by this device to rapidly assess stimulation effects on brain activity and illustrates how biomarkers such as these could be used clinically to select and titrate DBS parameters within therapeutic windows. In addition, the demonstration of sustained HC inhibition produced by local stimulation at relatively low levels in the awake animal is intriguing, given studies of this DBS modality to treat temporal lobe epilepsy [35-37]. 
Finally, the simultaneous recordings of thalamic and $\mathrm{HC}$ activity in free-roaming animals, under a variety of behavioral conditions, provided a window into the network dynamics between these two nodes of the Papez circuit. The ability to collect timed and triggered recordings offered a unique opportunity to sample brain activity throughout various times of the day, over long periods of time. The description and analyses of those recordings are beyond the scope of this manuscript; however, the examples shown in figures 7 and 8 provide a glimpse of the types of recordings that were obtained and clearly illustrate different states of this thalamohippocampal network. For example, the appearance of thalamic K complexes in both panels of figure 7a suggests that the animal was asleep. However, the dramatic difference in network activity between these two recordings indicates very different brain states and sleep stages, with panel $a_{2}$ likely reflecting slow-wave sleep, characterized by the presence of hippocampal SPW activity [38]. Figure 7b was a very commonly observed recording pattern, with strong theta bursting in the HC, but little corresponding thalamic activity. These two examples, along with the records in figure 8 , nicely demonstrate the two prominent firing patterns of hippocampal neural populations, theta and SPW, and the related status of the thalamus. During theta, which is associated with behavioral states where 'online' sensory information is processed and passed to the HC, the anterior thalamus appeared relatively inactive. In contrast, during SPW, considered to be an output state of the HC, when information was transferred back to cortical and sub-cortical structures [39], synchronous changes in thalamic activity were regularly observed.

The ability to detect specific patterns of LFP activity with the implanted device algorithms, as shown in figure 8, has clear clinical implications for potential use in patient monitoring (e.g. seizure tracking in epilepsy, beta band monitoring in Parkinson's disease) as well as expansion to future close-looped control of DBS devices in these and other indications.

In conclusion, this initial evaluation of a new hardware platform for chronic recording of neural activity in association with DBS therapies provides an encouraging first look at the performance and potential of this device. Given the fully implantable nature of the system, and the ability to concurrently stimulate and record from multiple locations within brain networks, this device holds promise to open new avenues of investigation of brain function, and offers the opportunity to study animal and human neurophysiology in a completely new '24/7' paradigm.

\section{Acknowledgement}

We wish to thank the following individuals for excellent surgical support and technical assistance: Drs. Linnea Lentz, Tina Billstrom, Randy Jensen, Ben Issacson, Justin Kemp.

\section{References}

1 Wichmann T, Delong MR: Deep-brain stimulation for basal ganglia disorders. Basal Ganglia 2011;1:65-77.

2 Lockman J, Fisher RS: Therapeutic brain stimulation for epilepsy. Neurol Clin 2009; 27:1031-1040.

-3 Greenberg BD, Gabriels LA, Malone DA Jr, Rezai AR, Friehs GM, Okun MS, Shapira NA, Foote KD, Cosyns PR, Kubu CS, Malloy PF, Salloway SP, Giftakis JE, Rise MT, Machado AG, Baker KB, Stypulkowski PH, Goodman WK, Rasmussen SA, Nuttin BJ: Deep brain stimulation of the ventral internal capsule/ventral striatum for obsessive-compulsive disorder: worldwide experience. $\mathrm{Mol}$ Psychiatry 2010;15:64-79.

4 Mallet L, Polosan M, Jaafari N, Baup N, Welter ML, Fontaine D, du Montcel ST, Yelnik J, Chéreau I, Arbus C, Raoul S, Aouizerate $\mathrm{B}$, Damier $\mathrm{P}$, Chabardès $\mathrm{S}$, Czernecki V, Ardouin C, Krebs MO, Bardinet E, Chaynes P, Burbaud P, Cornu P, Derost P, Bougerol T, Bataille B, Mattei V, Dormont D, Devaux
B, Vérin M, Houeto JL, Pollak P, Benabid AL, Agid Y, Krack P, Millet B, Pelissolo A, STOC Study Group: Subthalamic nucleus stimulation in severe obsessive-compulsive disorder. N Engl J Med 2008;359:21212134

5 Mayberg HS, Lozano AM, Voon V, McNeely HE, Seminowicz D, Hamani C, Schwalb JM, Kennedy SH: Deep brain stimulation for treatment-resistant depression. Neuron 2005;45:651-660.

-6 Malone DA Jr, Dougherty DD, Rezai AR, Carpenter LL, Friehs GM, Eskandar EN, Rauch SL, Rasmussen SA, Machado AG, Kubu CS, Tyrka AR, Price LH, Stypulkowski PH, Giftakis JE, Rise MT, Malloy PF, Salloway SP, Greenberg BD: Deep brain stimulation of the ventral capsule/ventral striatum for treatment-resistant depression. Biol Psychiatry 2009;65:267-275.

7 Laxton AW, Tang-Wai DF, McAndrews MP, Zumsteg D, Wennberg R, Keren R, Wherrett J, Naglie G, Hamani C, Smith GS, Lozano
AM: A phase I trial of deep brain stimulation of memory circuits in Alzheimer's disease. Ann Neurol 2010;68:521-534.

$\checkmark 8$ Buzsáki G, Draguhn A: Neuronal oscillations in cortical networks. Science 2004;304: 1926-1929.

-9 Fries P: A mechanism for cognitive dynamics: neuronal communication through neuronal coherence. Trends Cogn Sci 2005;9: 474-480.

10 Liebe S, Hoerzer GM, Logothetis NK, Rainer G: Theta coupling between V4 and prefrontal cortex predicts visual short-term memory performance. Nat Neurosci 2012;15:456462, S1-2.

11 Llinás RR, Ribary U, Jeanmonod D, Kronberg E, Mitra PP: Thalamocortical dysrhythmia: a neurological and neuropsychiatric syndrome characterized by magnetoencephalography. Proc Natl Acad Sci USA 1999;96: 15222-15227.

12 Brown P: Bad oscillations in Parkinson's disease. J Neural Transm Suppl 2006;27-30. 
13 Gatev P, Darbin O, Wichmann T: Oscillations in the basal ganglia under normal conditions and in movement disorders. Mov Disord 2006;21:1566-1577.

$\checkmark 14$ Brown P, Mazzone P, Oliviero A, Altibrandi MG, Pilato F, Tonali PA, Di Lazzaro V: Effects of stimulation of the subthalamic area on oscillatory pallidal activity in Parkinson's disease. Exp Neurol 2004;188:480-490.

$\checkmark 15$ Chen CC, Pogosyan A, Zrinzo LU, Tisch S, Limousin P, Ashkan K, Yousry T, Hariz MI, Brown P: Intra-operative recordings of local field potentials can help localize the subthalamic nucleus in Parkinson's disease surgery. Exp Neurol 2006;198:214-221.

$\checkmark 16$ Ince NF, Gupte A, Wichmann T, Ashe J, Henry T, Bebler M, Eberly L, Abosch A: Selection of optimal programming contacts based on local field potential recordings from subthalamic nucleus in patients with Parkinson's disease. Neurosurgery 2010;67: 390-397.

-17 Stanslaski S, Cong P, Carlson D, Santa W, Jensen R, Molnar G, Marks WJ Jr, Shafquat A, Denison T: An implantable bi-directional brain-machine interface system for chronic neuroprosthesis research. Conf Proc IEEE Eng Med Biol Soc 2009;2009:5494-5497.

-18 Stypulkowski PH, Giftakis JE, Billstrom TM: Development of a large animal model for investigation of deep brain stimulation for epilepsy. Stereotact Funct Neurosurg 2011;89:111-122.

19 Mirski MA, Ferrendelli JA: Interruption of the mammillothalamic tract prevents seizures in guinea pigs. Science 1984;226:7274.

20 Mirski MA, Fisher RA: Electrical stimulation of the mamillary nuclei raises seizure threshold to pentylenetetrazol in rats. Epilepsia 1994;35:1309-1316.

21 Mirski MA, Rossell LA, Fisher RA: Anticonvulsant effect of anterior thalamic high frequency electrical stimulation in the rat. Epilepsy Res 1997;28:89-100.
22 Hamani C, Ewerton FI, Bonilha SM, Ballester G, Mello LE, Lozano AM: Bilateral anterior thalamic nucleus lesions and high-frequency stimulation are protective against pilocarpine-induced seizures and status epilepticus. Neurosurgery 2004;54:191-195.

23 Hamani C, Hodaie M, Chiang J, del Campo M, Andrade DM, Sherman D, Mirski M, Mello LE, Lozano AM: Deep brain stimulation of the anterior nucleus of the thalamus: effects of electrical stimulation on pilocarpine-induced seizures and status epilepticus. Epilepsy Res 2008;78:117-123.

24 Hoadie M, Wennberg RA, Dostrovsky JO, Lozano AM: Chronic anterior thalamus stimulation for intractable epilepsy. Epilepsia 2002;43:603-608.

25 Kerrigan JF, Litt B, Fisher RS, Cranstoun S, French JA, Blum DE, Dichter M, Shetter A, Baltuch G, Jaggi J, Krone S, Brodie M, Rise M, Graves N: Electrical stimulation of the anterior nucleus of the thalamus for the treatment of intractable epilepsy. Epilepsia 2004;45:346-354.

26 Andrade DM, Zumsteg D, Hamani C, Hodaie M, Sarkissian S, Lozano AM, Wennberg RA: Long-term follow-up of patients with thalamic deep brain stimulation for epilepsy. Neurology 2006;66:1571-1573.

27 Osorio I, Overman J, Giftakis J, Wilkinson SB: High frequency thalamic stimulation for inoperable mesial temporal epilepsy. Epilepsia 2007;48:1561-1571.

28 Fisher R, Salanova V, Witt T, Worth R, Henry T, Gross R, Oommen K, Osorio I, Nazzaro J, Labar D, Kaplitt M, Sperling M, Sandok E, Neal J, Handforth A, Stern J, DeSalles A, Chung S, Shetter A, Bergen D, Bakay R, Henderson J, French J, Baltuch G, Rosenfeld W, Youkilis A, Marks W, Garcia P, Barbaro N, Fountain N, Bazil C, Goodman R, McKhann G, Babu Krishnamurthy K, Papavassiliou S, Epstein C, Pollard J, Tonder L, Grebin J, Coffey R, Graves N, SANTE Study Group: Electrical stimulation of the anterior nucleus of thalamus for treatment of refractory epilepsy. Epilepsia 2010;51:899-908.

29 Vanderwolf, CH, Cooley RK: The Sheep Brain: A Photographic Series, ed 2. London, AJ Kirby Co, 2002.
30 Holloway KL, Gaede SE, Starr PA, Rosenow JM, Ramakrishnan V, Henderson JM: Frameless stereotaxy using bone fiducial markers for deep brain stimulation. Neurosurgery 2005;103:404-413.

31 Wennberg R: Intracranial cortical localization of the human K-complex. Clin Neurophysiol 2010;121:1176-1186.

32 Charlet de Sauvage R, Lima da Costa D, Erre JP, Aran JM: Electrical and physiological changes during short-term and chronic electrical stimulation of the normal cochlea. Hear Res 1997;110:119-134.

33 Lesser RP, Lüders H, Klem G, Dinner DS, Morris $\mathrm{HH}$, Hahn J: Cortical afterdischarge and functional response thresholds: results of extraoperative testing. Epilepsia 1984;25: 615-621.

34 Blume WT, Jones DC, Pathak P: Properties of after-discharges from cortical electrical stimulation in focal epilepsies. Clin Neurophysiol 2004;115:982-989.

35 Velasco AL, Velasco M, Velasco F, Menes D, Gordon F, Rocha L, Briones M, Márquez I: Subacute and chronic electrical stimulation of the hippocampus on intractable temporal lobe seizures: preliminary report. Arch Med Res 2000;31:316-328.

>36 Vonck K, Boon P, Achten E, De Reuck J, Caemaert J: Long-term amygdalohippocampal stimulation for refractory temporal lobe epilepsy. Ann Neurol 2002;52:556-565.

-37 Cukiert A, Cukiert CM, Argentoni-Baldochi M, Baise C, Forster CR, Mello VA, Burattini JA, Lima AM: Intraoperative neurophysiological responses in epileptic patients submitted to hippocampal and thalamic deep brain stimulation. Seizure 2011;20:748-753.

38 Chrobak JJ, Sirota A, Buzsáki G: Hippocampal-neocortical dialog; in Stickgold R, Walker MP (eds): The Neuroscience of Sleep. Boston/Amsterdam, Academic Press/Elsevier, 2009, pp 37-43.

39 Buzsáki G, Chrobak JJ: Synaptic plasticity and self-organization in the hippocampus. Nat Neurosci 2005;8:1418-1420. 